\title{
CENTRALIDADE E CIDADES MÉDIAS: O SETOR DE SÁUDE EM MONTES CLAROS/MG
}

\author{
CENTRALITY AND MEDIUM CITIES: HEALTH SECTOR \\ IN MONTES CLAROS/MG
}

\author{
CENTRALIDAD Y MEDIANAS CIUDADES: EL SECTOR DE LA SALUD \\ EN MONTES CLAROS/MG
}

\author{
lara Soares de França - Universidade Estadual de Montes Claros - Montes Claros - Minas Gerais - Brasil \\ isfufu@yahoo.com.br \\ Beatriz Ribeiro Soares - Universidade Federal de Uberlândia - Uberlândia - Minas Gerais - Brasil \\ brsoares@ufu.br
}

\begin{abstract}
Resumo
As cidades médias passaram a despertar a atenção dos geógrafos brasileiros após a década de 1970 em estudos que abordavam as redes urbanas regionais, o padrão econômico, dentre outras características. Os primeiros estudos sobre cidades médias brasileiras estavam baseados no tamanho populacional para identificar quais seriam essas cidades. Por exemplo, o Instituto Brasileiro de Geografia e Estatística (IBGE) classifica como cidades médias os centros urbanos que possuem população entre 100 e 500 mil habitantes. Todavia, os estudos de autores como Amorim Filho, Bueno e Abreu (1982), Soares (1999), Sposito (2001) e Pereira e Lemos (2004) chamam a atenção para a necessidade de incorporação de outras variáveis nos estudos sobre as cidades médias, como a posição e importância na região na qual se encontram inseridas, as relações interurbanas e intraurbanas, a sua especialização e diversificação econômica, além da centralidade, entre outras. Montes Claros, cidade média do norte de Minas Gerais, possui características que a colocam numa posição de centro regional que comanda as áreas do seu entorno e os municípios com menor diversidade de funções. Nesse sentido, este artigo analisa a centralidade que esta cidade média exerce nos municípios do seu entorno considerando o setor de saúde.
\end{abstract}

Palavras-chave: cidade média, centralidade, setor de saúde, Montes Claros/MG.

\begin{abstract}
Medium cities began to arouse the attention of Brazilian geographers after the 1970's in studies on regional urban networks, economic pattern among other features. The first studies on Brazilian medium cities were based on population size parameter in order to identify those cities. For instance, the Instituto Brasileiro de Geografia e Estatística (IBGE) which classifies as medium cities those urban centers with population from 100 to 500 thousand inhabitants. However, Amorim Filho, Bueno e Abreu (1982), Soares (1999), Sposito (2001) and Pereira \& Lemos (2004) among others highlight the need for incorporation of other variables in the studies regarding medium cities as its position and importance in the region where it is located, the inter-urban and intraurban factors, its specialization and economic diversification besides centrality. Montes Claros, a medium city in the North of Minas Gerais, displays characteristics that put it in a position of regional center which commands the surrounding areas and the cities with lower diversity of functions. Thus, this paper analyzes the centrality role that this medium city plays on its surrounding cities considering the health sector.
\end{abstract}

Key words: medium city, centrality, health sector, Montes Claros/MG. 


\section{Resumen}

Las ciudades medianas pasaron a despertar la atención de los geógrafos brasileños después de la década de 1970 en estudios que abordaban las redes urbanas regionales, el padrón económico, además de otras características. Los primeros estudios sobre ciudades medianas brasileñas estaban basados en el parámetro tamaño de la población para identificar cuáles serían estas ciudades. Por ejemplo, el Instituto Brasileño de Geografía y Estadística (IBGE) clasifica como ciudades medianas aquellos centros urbanos que posen población entre 100 y 500 mil habitantes. Todavía, los estudios de Amorim Filho, Bueno e Abreu (1982), Soares (1999), Sposito (2001) y Pereira e Lemos (2004) entre otros, llaman La atención para la necesidad de la incorporación de otras variables en los estudios sobre las ciudades medianas, como su ubicación e importancia en la región en la cual encuéntrense inseridas, las relaciones interurbanos e intraurbanos, su especialización y diversificación económica, además de la centralidad, entre otros. Montes Claros, ciudad mediana ubicada en norte de Minas Gerais, posee características que la ponen en una posición de centro regional que comanda las áreas de su entorno y los municipios con menor diversidad de funciones. Así siendo, este artículo analiza la centralidad que esta cuidad mediana ejerce en los municipios a su alrededor considerando el sector de la salud.

Palabras clave: ciudad mediana, centralidad, industria de la salud, Montes Claros/MG.

\section{Introdução}

Nos estudos sobre redes urbanas, aglomerações urbanas e regiões de influência, as cidades são analisadas, entre outros fatores, de acordo com as relações que estabelecem entre si, sejam elas de trocas diretas ou indiretas, relações de dependência, subordinação, polarização ou complementaridade. É a centralidade que as cidades exercem que define o importante papel que desempenham.

Ao formular a Teoria das localidades centrais ou da centralidade, na década de 1930, Christaller (1966) incorporou os conceitos de localidades centrais e de centralidade. Nessa teoria "os centros são denominados localidades centrais e a centralidade de que dispõem é derivada de seu papel como centros distribuidores de bens e serviços, ou seja, das funções centrais que desempenham" (IBGE, 1987, p. 11).

Para Raffestin (1993, p. 187), "a centralidade é, portanto, na sua essência, uma entidade com duas faces: um ‘topos' e uma ‘tensão”. Topos e tensão que persistem enquanto estiverem ligados, e que, dinamicamente, se traduzem por movimentos centrípetos ou centrífugos.

A centralidade urbana materializa-se em razão das trocas e das interações realizadas entre as cidades que, dessa forma, estão conectadas a uma rede urbana. Cada cidade é singular, diferente, histórica, econômica e socialmente; assim, as trocas entre elas são espacialmente desiguais em virtude da oferta de bens e serviços que as dotam, ou não, de centralidade. Se há graus diferentes de especialização das cidades no território, a sua atuação na rede é também diferente. Há aquelas que possuem maior im- 
portância na rede urbana e outras que interagem e se comunicam com os centros mais dinâmicos da rede, buscando bens e serviços necessários à reprodução da vida.

Whitacker (2007) propõe que, para compreender a constituição da centralidade, é preciso considerar, sobretudo, os fluxos: "[...] são os fluxos os elementos determinantes, muito mais que a localização. Esses fluxos são incrementados pelas comunicações e telecomunicações que são traduzidas em trocas, decisões, gestão, controle e irradiação de valores” (p.1).

Sendo assim, a posição hierárquica de uma cidade na rede em que se insere resulta de seu perfil econômico, associado à concentração de atividades produtivas, riquezas, população, infraestrutura, serviços alocados e, especialmente, de sua capacidade de absorção de investimentos públicos e privados. A concentração espacial desses elementos fomenta as relações entre as cidades; além disso, eles podem definir a polarização a favor das metrópoles e das cidades médias, a subordinação de centros de menor dinamismo e, ainda, a competição entre as cidades da rede.

A centralidade resulta da capacidade de polarização de alguns centros nos espaços em que se inserem. Ela se expressa por meio da diversidade e da especialização em bens e serviços do centro urbano principal. Assim, quanto maior a oferta desses serviços, maior será o seu grau de centralidade.

Nesse sentido, a análise de Montes Claros/MG como cidade média está vinculada, dentre outros elementos, à sua centralidade como cidade polo, ou seja, o papel que exerce nos municípios de seu entorno, construída através de fatores diversos, dentre eles, o setor de saúde, objeto de estudo deste artigo.

Para isso, realizou-se o levantamento bibliográfico de estudos a respeito das cidades médias e centralidades urbanas e também de informações sobre a infraestrutura do setor de saúde dos municípios.

Em relação à pesquisa documental, diagnosticou-se o número de atendimentos da população dos municípios limítrofes nos principais hospitais de Montes Claros em 2010: Aroldo Tourinho, Universitário Clemente de Faria e Santa Casa da Misericórdia. Esses dados foram disponibilizados pela administração dos hospitais por meio do Serviço de Atendimento Médico e Estatística (Same), com consultas neste sistema, no período de janeiro a maio de 2011. 
Foram feitas, ainda, entrevistas a gestores dos municípios do entorno sobre os fatores que motivam o fluxo da população para Montes Claros, destacando, nesse sentido, o setor de saúde.

Montes Claros/MG é apontada em estudos de autores como Andrade e Lodder, 1979; Amorim Filho, Bueno e Abreu, 1982; Pereira e Lemos, 2004; Pereira, 2007; França, 2012, 2007, como uma cidade média na região Norte de Minas. Ela exerce polarização e atração regional por concentrar diversas atividades econômicas e prestação de serviços, além da infraestrutura que possui. Dentre as atividades e os serviços presentes nessa cidade, que a credenciam como lócus regional, podem ser destacados o comércio diversificado de produtos (atacado e varejo) e o serviço especializado de saúde que, em razão do planejamento estadual e federal, tem uma amplitude regional. O serviço de educação superior público e privado atrai pessoas de diversas cidades norte mineiras, das regiões central, noroeste e nordeste de Minas, além do sul da Bahia.

Vale ressaltar que Montes Claros, cidade média, vivencia profundas transformações urbanas que refletem no seu alcance regional e as atividades ancoradas no setor terciário (comércio e prestação de serviços) e no industrial se expandem. Isso porque a cidade ampliou a sua rede de estabelecimentos comerciais varejistas e atacadistas, o que demonstra mais especialização e diversificação do setor terciário; os setores de saúde e de educação superior, notadamente, se ampliam e se diversificam; e a atividade industrial passa por processos de modernização, pelo uso de novas tecnologias, resultantes de demandas de novos ramos industriais instalados em Montes Claros.

Verificam-se, ainda, grandes investimentos direcionados ao setor imobiliário da cidade e à construção civil, com o intuito de atender à demanda de consumo e/ou habitação das classes de alta, média e baixa renda. Os recursos advindos desses setores, para a construção de condomínios fechados e conjuntos habitacionais ou habitações populares, ${ }^{1}$ impulsionam a expansão urbana, vinculada ao processo de acumulação e de reprodução do capital.

Trata-se de uma reprodução socioespacial e econômica característica da atual fase de urbanização vivenciada por Montes Claros, o que confirma a sua centralidade no norte de Minas, por reunir funções econômicas, políticas e de infraestrutura importantes, induzindo o fluxo de pessoas, mercadorias, bens e capitais. 
Conforme Whitacker (2007, p. 1), "a dinâmica de concentração e dispersão cria e recria centralidades que irão ocupar e valorar diferentemente e diferencialmente territórios no tecido urbano e na dimensão da rede urbana e se traduzem em segmentação de usos e não usos e na fragmentação socioespacial."

A articulação ou as trocas estabelecidas entre as cidades podem ser interpretadas através da centralidade, isto é, a função de destaque de um centro urbano em sua região de influência. As cidades são localidades centrais que exercem a centralidade; nesse caso, a centralidade urbana. A análise da centralidade urbana $^{2}$ é fundamental nas pesquisas sobre as interações socioespaciais entre cidades.

\section{A centralidade da cidade média de Montes Claros}

O município de Montes Claros localiza-se no norte do Estado de Minas Gerais e possui uma população total de 361.971 habitantes (IBGE, 2010). Nas últimas décadas, esta cidade média passou por um significativo incremento na sua taxa de população urbana que evoluiu de $73 \% \mathrm{em}$ 1970, para 95\% em 2010, uma média maior do que a nacional.

A partir dos investimentos oriundos da Superintendência de Desenvolvimento do Nordeste (Sudene, 1959) e do Programa Nacional de Apoio às Capitais e Cidades de Porte Médio (PNCCPM, 1975), bem como de capitais públicos e privados, observam-se mudanças na paisagem, estrutura intra e interurbana de Montes Claros/MG. Tem-se um período de expansão urbana, instalação de novas indústrias na cidade e, com isso, grande dinamização econômica e estrutural.

Montes Claros/MG apresenta uma das maiores economias mineiras e, em 2010, já ocupava o $11^{\circ}$ lugar no ranking municipal de composição do Produto Interno Bruto (PIB) estadual com o valor de R\$ 4.501.662.000,00; considerando o PIB por setores econômicos, no ano de 2010, destaca-se a participação do setor de serviços com 74\%, enquanto o setor industrial e o agropecuário responderam, respectivamente, por 24\% e 3\% do PIB municipal (IBGE - Cidades, 2013).

Ao analisar o PIB de Montes Claros/MG por setores da economia, pode-se afirmar que a expansão do setor terciário, especialmente a educação superior e o setor de saúde, na última década, é um dos principais vetores do crescimento econômico no PIB de serviços e da riqueza da ci- 
dade como um todo. O desenvolvimento do setor terciário é uma tendência marcante na dinâmica econômica das cidades médias, como é o caso da cidade em foco.

Sobre o provimento de infraestrutura e de equipamentos urbanos em cidades médias, Sanfeliu e Torné (2004, p. 16) afirmam:

Básicamente son los diferentes contextos socioeconómicos y económicos los que explican las diferencias existentes en los niveles de infraestructura y equipamiento urbano. Pero los datos que comentamos en este apartado muestran también el rol y capitalidad que las ciudades juegan en su propio territorio, sobre todo en el tema de equipamientos urbanos. Así, por ejemplo, ciudades con un destacado papel regional concentran un buen número de equipamientos especializados (universidades, hospitales, centros deportivos, bibliotecas), equipamiento que han de dar cobertura a amplios territorios del área de influencia del núcleo.

Pode-se inferir que quanto maior for a capacidade de oferta de bens e serviços de uma cidade, maior será a sua centralidade. Dessa forma, a relevância da cidade média de Montes Claros/MG, no norte de Minas, deve-se a vários fatores, tais como a estrutura e a diversidade de bens, comércios e prestação de serviços existentes. A cidade é um polo atrativo para atender às necessidades da população local e regional, destacando-se no setor de saúde:

O espaço de saúde, na cidade de Montes Claros/MG, apresenta um grande destaque, devido à organização e à existência de serviços médicos especializados e uma rede de hospitais e clínicas interligadas, sendo referência regional. É possível identificar sedes de empresas de seguro saúde, seguindo a lógica nacional do sistema de saúde privado. (Pereira, 2007, p. 140)

Montes Claros/MG oferece uma diversificada e complexa infraestrutura dos serviços de saúde. ${ }^{3}$ Para se beneficiar desses serviços, uma grande quantidade de pessoas de outros municípios se desloca para Montes Claros/MG. O transporte de pacientes é realizado em ambulâncias, táxis, veículos próprios ou fretados e, especialmente, em micro-ônibus de Consórcios Intermunicipais de Saúde (CIS).

Próximo dos grandes hospitais, há um conjunto de unidades complementares: clínicas especializadas, consultórios médicos e odontológicos, unidades de fisioterapia e laboratórios. Além disso, existe uma rede de serviços destinados à população que procura atendimento de saúde 
como farmácias, drogarias, lanchonetes, restaurantes, pensões, dentre outros. Nesse sentido, configuram-se verdadeiros territórios da saúde; os serviços prestados nesses locais e os estabelecimentos comerciais ali estabelecidos movimentam a economia da cidade.

Os fluxos no setor de saúde entre Montes Claros e os municípios do entorno

De acordo com o estudo feito, foram constatados os fluxos estabelecidos no setor de saúde entre a cidade média de Montes Claros e estes municípios do entorno: Bocaiúva, Coração de Jesus, São João da Ponte, Francisco Sá, Capitão Enéas, Mirabela, Claro dos Poções, Patis, São João da Lagoa, Juramento e Glaucilândia. Para isso, foram realizadas entrevistas com os representantes do poder público municipal (Secretários de planejamento, Chefes de gabinete ou Assessores) de cada município do entorno com o objetivo de identificar as relações estabelecidas entre Montes Claros e os demais municípios nas diversas áreas. Segundo os entrevistados, as principais relações são estabelecidas nas áreas de saúde, educação, trabalho e comércio, conforme pode ser observado na Figura 1:

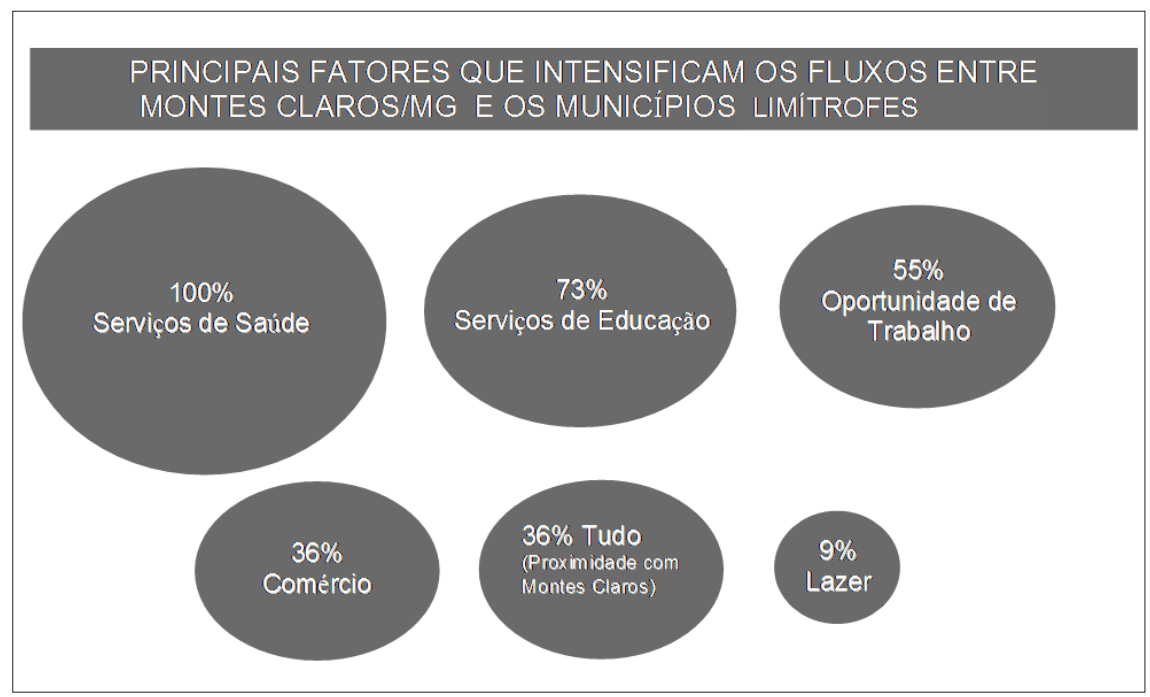

Figura 1: Principais fatores que intensificam os fluxos entre Montes Claros e os municípios do entorno, 2011.

Fonte: Pesquisa Direta, 2011

Org.: França, I. S. de; 2011. 
Como a proximidade entre as cidades foi apontada por muitos entrevistados como o principal motivo para o estabelecimento das relações entre os municípios vizinhos e Montes Claros, observa-se que as distâncias intermunicipais reforçam os fluxos ali existentes, sobretudo entre Montes Claros e os municípios mais próximos e frágeis economicamente, como é o caso de Juramento, Glaucilândia e São João da Lagoa.

Para compreender como é a organização do serviço de saúde médico-hospitalar especializado dos municípios limítrofes a Montes Claros, foi solicitada essa informação ao representante da prefeitura.

Com as informações obtidas, verificou-se que há poucos estabelecimentos de saúde nesses municípios. Apenas em Bocaiúva, Francisco Sá, Coração de Jesus, Capitão Enéas e Mirabela existe hospital municipal; em São João da Ponte, há uma Fundação de Saúde; em todos os municípios da aglomeração, há postos de saúde com atendimentos menos complexos, preventivos e, além disso, desenvolvem o Programa de Saúde da Família (PSF), há também serviços farmacêuticos e odontológicos públicos e particulares, com exceção de Glaucilândia, visto que é o único município que não possui laboratório de análises clínicas. No Quadro 1, encontram-se os números referentes a essas questões.

Quadro 1: Infraestrutura de saúde instalada nos municípios, 2011.

\begin{tabular}{|l|c|c|c|c|}
\hline Município & \multicolumn{5}{|c|}{ Estabelecimento/Hierarquia } \\
\hline Aásica & $\begin{array}{c}\text { Média } \\
\text { Complexidade }\end{array}$ & $\begin{array}{c}\text { Alta Complexidade } \\
\text { Ambulatorial } \\
\text { (Habilitado) }\end{array}$ & $\begin{array}{c}\text { Alta Complexidade } \\
\text { Hospitalar } \\
\text { (Habilitado) }\end{array}$ \\
\hline Montes Claros & 110 & 258 & 68 & 6 \\
\hline Bocaiúva & 16 & 17 & 1 & 1 \\
\hline Capitão Enéas & 12 & 4 & 2 & 1 \\
\hline Coração de Jesus & 8 & 6 & 1 & 1 \\
\hline Mirabela & 6 & 2 & 1 & 1 \\
\hline Francisco Sá & 12 & 4 & 1 & 1 \\
\hline São João da Ponte & 14 & 3 & 1 & 0 \\
\hline Claro dos Poções & 6 & 3 & 0 & 0 \\
\hline Juramento & 4 & 0 & 1 & 0 \\
\hline Patis & 1 & 1 & 0 & 0 \\
\hline São João da Lagoa & 2 & 1 & 0 & 0 \\
\hline Glaucilândia & 5 & 1 & & \\
\hline
\end{tabular}


Assim, conclui-se que o atendimento médico-hospitalar realizado nesses municípios é de baixa ou média complexidade. O setor de saúde de Montes Claros se distingue daquele dos municípios do entorno pela quantidade, variedade e, sobretudo, pelas especialidades médicas disponíveis. Por isso, muitas pessoas dos municípios vizinhos que não dispõem desses serviços buscam atendimento em Montes Claros, o que ratifica seu forte raio de atração no setor de saúde. Ao realizarem parcerias com Montes Claros para atendimento, especialmente, de média e alta complexidade, os municípios se tornam altamente dependentes da pactuação com Montes Claros para atender à população nos três principais hospitais da cidade: Santa Casa de Misericórdia, Hospital Universitário Clemente de Faria e Hospital Aroldo Tourinho.

Nesse sentido, outra forma de averiguar a centralidade de Montes Claros foi através do número de atendimentos da população dos municípios vizinhos nos hospitais da cidade. Para essa análise, realizou-se uma pesquisa sobre o registro de atendimento de pacientes em 2010, nos três principais hospitais da cidade (Foto 1).

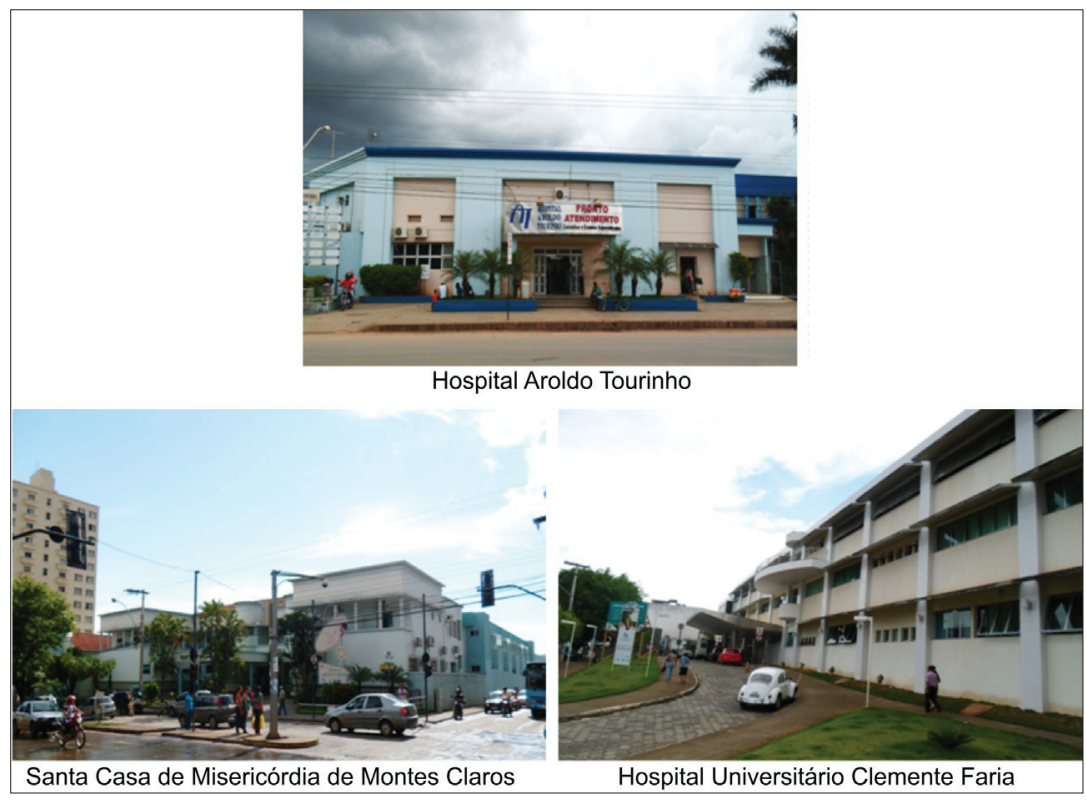

Foto 1: Hospitais, 2013.

Autor: França, I. S. de; 2013. 
Essas informações são referentes aos onze municípios do entorno, com exceção de Montes Claros. Os referidos dados foram disponibilizados pela administração dos hospitais através do Same e o número de atendimentos de pacientes dos municípios da aglomeração foi agrupado considerando a base de dados disponível nos hospitais. Vale ressaltar que o registro dessa informação sobre os hospitais, no Same, refere-se ao local de origem do paciente, ou seja, sua procedência, e não o local de nascimento. Dessa forma, esses dados atendem às finalidades deste artigo, uma vez que se referem ao deslocamento da população dos pequenos municípios do entorno para procurar atendimento médico-hospitalar em Montes Claros.

Em 2010, Bocaiúva, Claro dos Poções, Coração de Jesus e Capitão Enéas foram as cidades do entorno com maior número de atendimentos nos hospitais de Montes Claros, correspondendo, respectivamente: 23\%, 15\%, 12\% e 11\% de um total de 16.035 atendimentos naquele ano (Gráfico 1).

Gráfico 1: Municípios do entorno de Montes Claros/MG: atendimento nos três principais hospitais de Montes Claros, 2010.

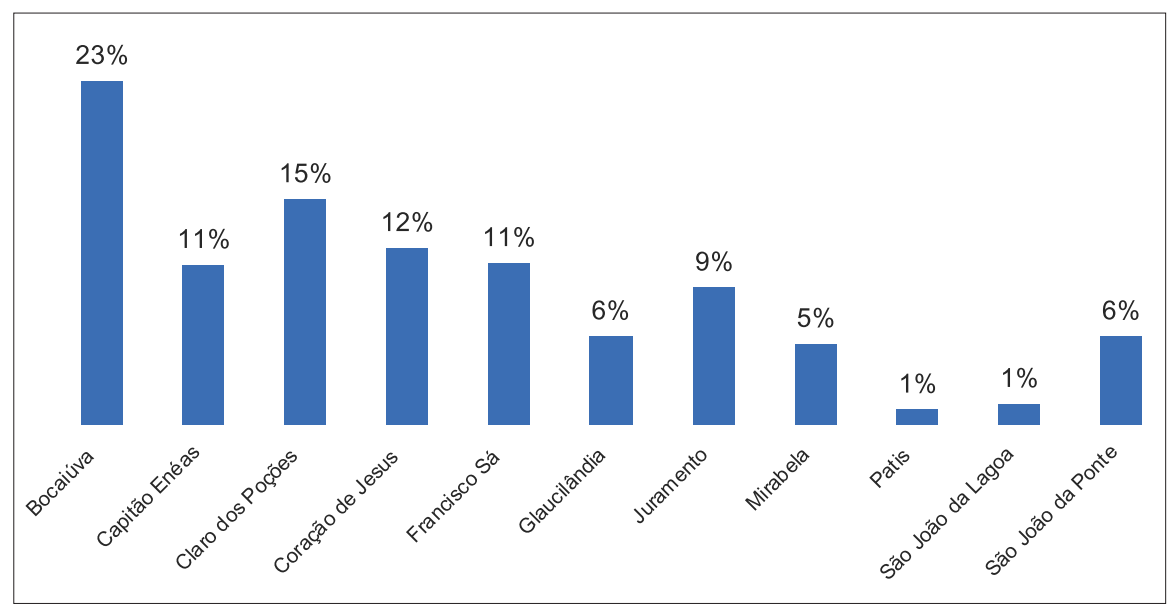

Fonte: SAME, Hospitais Aroldo Tourinho, HUCF e Santa Casa, 2010.

Org.: França, I. S. de; 2011. 
Mapa 1: Atendimento nos três principais hospitais de Montes Claros/MG, 2010.

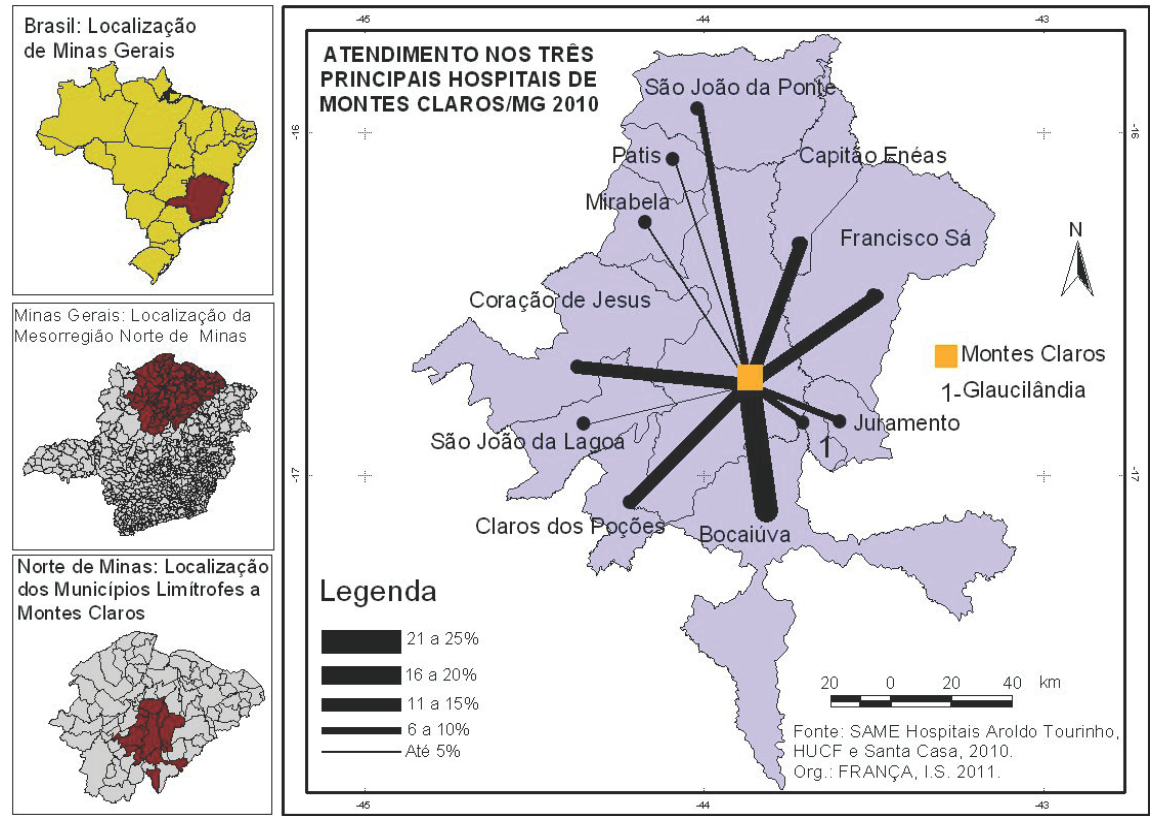

O atendimento de pessoas dos municípios limítrofes a Montes Claros corresponde a aproximadamente $5 \%$ do atendimento total geral nos três principais hospitais dessa cidade que, em 2010, foi equivalente a 358.319. Desse total, 52.720 (14,72\%) referem-se ao atendimento de pessoas provenientes de todas as cidades do norte de Minas, o que revela também a grande procura de serviços de saúde no âmbito regional na cidade média de Montes Claros.

\section{Considerações finais}

Este artigo analisou a centralidade econômica de Montes Claros na região Norte de Minas, uma vez que se destaca pela infraestrutura em serviços de saúde. Dessa forma, verificou-se que há uma forte relação de dependência entre os municípios do entorno e a cidade; por esse motivo, constantemente há grande fluxo de pessoas ali procurando atendimento médico-hospitalar, isto é, as pessoas buscam serviços especializados em 
consultas, exames, cirurgias e tratamentos médicos em geral. Como meio de transporte, elas utilizam ambulâncias, táxis, veículos próprios ou fretados e micro-ônibus de consórcios regionais de saúde.

Os resultados aqui expostos demonstram a dificuldade de integração do planejamento público intermunicipal a partir da cidade média de Montes Claros. Há uma grande diversidade socioeconômica entre os municípios do entorno (pequenas cidades) e Montes Claros (cidade média). A estrutura econômica que os pequenos municípios em estudo dispõem ratifica esse argumento e consequentemente reforça a centralidade de Montes Claros, posto que a população desses municípios procura o atendimento especializado nos setores de saúde, educação e comércio em Montes Claros. As demandas básicas ou de primeira necessidade (produtos, serviços, bens e mercadorias) são atendidas no próprio município. A não ser os CIS e a própria hierarquização e regionalização do SUS, não há nos municípios limítrofes a Montes Claros outras ações ou propostas de gestão pública intermunicipal ou regional. Todavia, as intensas relações estabelecidas entre a população desses municípios em diversos setores, tais como a saúde aqui em análise, a revelia do poder público, revelam a necessidade de políticas de integração intermunicipal e regional assentadas na diversidade e especificidade de cada município visando à melhoria da qualidade de vida de todos os cidadãos.

\section{Notas}

1. Uma análise do processo de expansão urbana vertical e horizontal em Montes Claros/MG está sendo desenvolvida com a pesquisa Novas formas de consumo residencial e comercial e a dinâmica espacial da cidade média de Montes Claros: o processo de verticalização e os condomínios horizontais após a década de 1990 (França; Almeida, 2012), de acordo com a Resolução n. 34 CEPEx/2012.

2. Outros autores também contribuem com a temática centralidades urbanas, destacando-se: Lefebvre (1999) , Hall (2006) e Ribeiro Filho; Souza (2009).

3. De acordo com o site do DATASUS-CNES (2011), Montes Claros/MG possui 938 leitos, em 436 estabelecimentos de saúde divididos em 6 níveis de hierarquia. Desse total, 281 são privados e 151, públicos; 144 pertencem à rede municipal e 7, à rede estadual. O município conta com um total de 88 centros de saúde ou unidades básicas de saúde nos bairros da cidade e na zona rural. Há uma diversidade de serviços especializados para tratamentos de alta complexidade. Dentre os seis hospitais gerais de atendimento pelo Sistema Único de Saúde (SUS), destacam-se: Santa Casa de Misericórdia, Hospital Universitário Clemente de Faria, Hospital Aroldo Tourinho e Hospital Dilson Godinho. Além destes, outros diversos serviços são encontrados na cidade como, por exemplo, 
a sede regional do Serviço de Atendimento Móvel Urgente (SAMU) e o Hemocentro Regional de Montes Claros/MG (Hemominas), o que reforça o papel da cidade como polarizadora dos serviços de saúde na mesorregião.

\section{Agradecimento}

Fundação de Amparo à Pesquisa do Estado de Minas Gerais (FAPEMIG).

\section{Referências}

AMORIM FILHO, Oswaldo Bueno; BUENO, Maria Elizabeth Taitson; ABREU, João Figueiredo. Cidades de porte médio e o programa de ações sócio-educativoculturais para as populações carentes do meio urbano em Minas Gerais. Boletim de Geografia Teorética, Rio Claro/SP, v. 2, n. 23-24, p. 33-46, 1982.

ANDRADE, Thompson; LODDER, Celsius. A. Sistema urbano e cidades médias no Brasil. IPEA. Rio de Janeiro: IPEA/INPES, 1979.

BRASIL. Lei $n^{\circ} 3.692$ de 15 de dezembro de 1959 cria a Superintendência de Desenvolvimento do Nordeste/Sudene. Disponível em: <http://www.planalto.gov. br/>. Acesso em: 2 jan. 2010.

CHRISTALLER, Walter. Teoria das localidades centrais. In: . Central places in southern germany. Englewood Cliffs: Prentice-Hall, 1966.

COLEGIADO de SECRETÁRIOS EXECUTIVOS dos CONSÓRCIOS INTERMUNICIPAIS de SAÚDE de MINAS GERAIS/COSECS. Disponível em: <http://www.cosecsmg.org.br/COSECS2003/index_geral.htm >. Acesso em: 16 ago. 2009.

DATASUS. Disponível em: <http://cnes.datasus.gov.br/Mod_Ind_Tipo_Leito. asp?VEstado=31\&VMun=314330htm $>$. Acesso em: 6 ago. 2009.

FRANÇA, Iara Soares de. A cidade média e suas centralidades: o exemplo de Montes Claros no Norte de Minas Gerais. Dissertação (Mestrado em Geografia) - Instituto de Geografia, Universidade Federal de Uberlândia/UFU, Uberlândia, 2007. 208p.

FRANÇA, Iara Soares de. Aglomeração urbana descontínua de Montes Claros/MG: novas configurações socioespaciais. Tese (Doutorado em Geografia) - Instituto de Geografia, Universidade Federal de Uberlândia/UFU, Uberlândia, 2012. $393 f$.

HALL, Tim. Urban geography. 3rd Ed. P.cm. (Routledge contemporary human geography series). 2006. 200p.

IBGE. Instituto Brasileiro de Geografia e Estatística. Região de influência das cidades/REGIC. Rio de Janeiro, 1987.

IBGE. Instituto Brasileiro de Geografia e Estatística.). Censo Demográfico. 2010. Disponível em: <http://www.ibge.gov.br htm/>. Acesso em: 12 jun. 2011. 
IBGE. Instituto Brasileiro de Geografia e Estatística (IBGE - Cidades). Produto Interno Bruto dos Municípios/PIB. 2013.

LEFEBVRE, Henri. A revolução urbana. Tradução de Sergio Martins. Belo Horizonte: Ed. UFMG, 1999.

PEREIRA, Anete Marília. Cidade média e região: o significado de Montes Claros no norte de Minas Gerais. Tese (Doutorado) - Instituto de Geografia, Universidade Federal de Uberlândia, Uberlândia, 2007.

PEREIRA, Fabiano Maia; LEMOS, Mauro Borges. Cidades médias: uma visão nacional e regional. Anais do XI Seminário sobre economia mineira. Diamantina, 24 a 27 de agosto de 2004. Disponível em: <http:॥.www.cedeplar.ufmg.br>. Acesso em: 29 abr. 2010.

Programa Nacional de Apoio às Capitais e Cidades de Porte Médio/ PNCCPM. 1975. Disponível em: <http:॥.www.planejamento.gov.br/>. Acesso em: 15 jan. 2006.

RAFFESTIN, Claude. Por uma geografia do poder. São Paulo: Ática, 1993.

RIBEIRO FILHO, Vitor; SOUZA, Marcus Vinicius Mariano de. O surgimento de novas centralidades nas cidades médias: análise comparativa entre os subcentros Luizote de Freitas e São Jorge em Uberlândia/MG, 2009.

SANFELIU, C. B.; TORNÉ, J. M. Miradas a otros espacios urbanos: las ciudades intermédias. Revista Electrónica de Geografia y Ciências Sociales, Universidad de Barcelona. ISSN: 1138-9788. Depósito Legal: B. 21.741-98 v. VIII, n. 165, 15 de mayo de 2004.

SOARES, B. R. Repensando as cidades médias brasileiras no contexto da globalização. Formação, Presidente Prudente: Ed. Unesp, n. 6, p. 55-64, 1999.

SPOSITO, Maria Encarnação Beltrão. As cidades médias e os contextos econômicos contemporâneos. In: SPOSITO, Maria Encarnação Beltrão (Org.). Urbanização e cidades: perspectivas geográficas. Presidente Prudente: (s.n.), 2001.

WHITACKER, Arthur Magon. Inovações tecnológicas, mudanças nos padrões locacionais e na configuração da centralidade em cidades médias. Anais do X Coloquio Internacional de Geocrítica: los problemas del mundo actual. Soluciones Y Alternativas desde la Geografía y las Ciencias Sociales. Porto Alegre, 28 de mayo, 1 de junio de 2007. Universidade Federal do Rio Grande do Sul.

\section{Sites consultados}

http:॥www.datasus.gov.br

http:॥www.fjp.gov.br

http:॥www.ibgecidades.gov.br 
lara Soares de França - Possui Graduação em Geografia pela Universidade Estadual de Montes Claros, Mestrado e Doutorado em Geografia pela Universidade Federal de Uberlândia. Atualmente é professora do Departamento de Geociências da Universidade Estadual de Montes Claros e bolsista pela Fundação de Amparo à Pesquisa do Estado de Minas Gerais.

Beatriz Ribeiro Soares - Possui Graduação em Geografia pela Universidade Federal de Uberlândia, Mestrado e Doutorado em Geografia pela Universidade de São Paulo. Atualmente é professora da Universidade Federal de Uberlândia.

Recebido para publicação em 14 de novembro de 2013 Aceito para publicação em 19 de janeiro de 2014 